BULLETIN OF THE

AMERICAN MATHEMATICAL SOCIETY

Volume 77, Number 6, November 1971

\title{
WEIGHTED APPROXIMATION OF CONTINUOUS FUNCTIONS ${ }^{1}$
}

\author{
BY JOÃO B. PROLLA
}

Communicated by Felix Browder, June 17, 1971

1. Notation. Let $X$ be a completely regular Hausdorff space and $E$ a (real or complex) locally convex Hausdorff space. $F(X, E)$ is the vector space of all mappings from $X$ into $E$, and $C(X, E)$ is the vector subspace of all such mappings that are continuous. $B_{\infty}(X, E)$ is the vector subspace of $F(X, E)$ consisting of those bounded $f$ that vanish at infinity. The vector subspace $C(X, E) \cap B_{\infty}(X, E)$ is denoted by $C_{\infty}(X, E)$. If $X$ is locally compact, $\mathfrak{K}(X, E)$ will denote the subspace of $C(X, E)$ consisting of those functions that have compact support. The corresponding spaces for $E=\boldsymbol{R}$ or $\boldsymbol{C}$ are written omitting $E$. A weight $v$ on $X$ is a nonnegative upper semicontinuous function on $X$. A directed family of weights on $X$ is a set of weights on $X$ such that given $u, v \in V$ and $\lambda \geqq 0$ there is a $w \in W$ such that $\lambda u, \lambda v \leqq w$. If $U$ and $V$ are two directed families of weights on $X$ and for every $u \in U$ there is a $v \in V$ such that $u \leqq v$, we write $U \leqq V$. If $V$ is a directed family of weights on $X$, the vector space of all $f \in F(X, E)$ such that $v f \in B_{\infty}(X, E)$, for any $v \in V$, is denoted by $F V_{\infty}(X, E)$ and is called a weighted function space. On $F V_{\infty}(X, E)$ we shall consider the topology determined by all the seminorms $f \mapsto \sup \{v(x) p(f(x)) ; x \in X\}$ where $v \in V$ and $p$ is a continuous seminorm on $E$. $C V_{\infty}(X, E)$ will denote the subspace $F V_{\infty}(X, E) \cap C(X, E)$, equipped with the induced topology. The weighted function spaces $C V_{\infty}(X, E)$ will be called Nachbin spaces.

2. Completeness properties of Nachbin spaces [6]. If for every $x \in X$ there is a weight $u \in U$ such that $u(x)>0$, we write $U>0$.

Lemma. If $E$ is complete and $U>0$, then $F U_{\infty}(X, E)$ is complete.

Theorem 1. Suppose that $E$ is complete, and $U$ and $V$ are two directed families of weights on $X$ with $U \leqq V$. If $V>0$ on $X$ and $C U_{\infty}(X, E)$ is closed in $F U_{\infty}(X, E)$, the Nachbin space $C V_{\infty}(X, E)$ is complete.

AMS 1970 subject classifications. Primary 46E10, 46E40; Secondary 46G10.

Key words and phrases. Continuous vector-valued functions, weights, Nachbin spaces, completeness, vector-valued bounded Radon measures, Bishop's generalized Stone-Weierstrass theorem, tensor product of topological modules.

1 This research was supported in part by NSF Grant GP-22713. 
In case $E$ is $R$ or $C$, the above theorem was obtained by Summers, under the hypothesis that $U>0$ on $X$. (See Theorem 3.6 of [10].)

Theorem 2. Suppose that $E$ is complete and $U$ and $V$ are two directed families of weights on $X$ with $U \leqq V$. If $V>0$ on $X$ and $C U_{\infty}(X, E)$ is quasi-complete, the Nachbin space $C V_{\infty}(X, E)$ is quasicomplete.

3. Dual spaces [6]. Throughout this paragraph $X$ will be a locally compact Hausdorff space. In this case, for any set of weights $V$ on $X$, the space $\mathscr{K}(X, E)$ is densely contained in $C V_{\infty}(X, E)$. In fact, even $\mathscr{K}(X) \otimes E$ is densely contained in $C V_{\infty}(X, E)$. Let $E_{w}^{\prime}$ denote the topological dual of $E$ endowed with the topology $\sigma\left(E^{\prime}, E\right)$. An $E_{v}^{\prime}$-valued bounded Radon measure $u$ on $X$ is a continuous linear mapping $u$ from $\mathfrak{K}(X)$ into $E_{w}^{\prime}$ when $\mathscr{K}(X)$ is endowed with the topology of uniform convergence on $X$. Following Grothendieck [4], an $E_{w}^{\prime}$-valued bounded Radon measure $u$ on $X$ is called integral if the linear form $L$ defined over $\Re(X) \otimes E$ by $L\left(\sum \phi_{i} \otimes y_{i}\right)=\sum\left\langle y_{i}, u\left(\phi_{i}\right)\right\rangle$ is continuous in the topology induced by $C_{\infty}(X, E)$, in which case it can be uniquely continuously extended to $C_{\infty}(X, E)$. Let $L \in C_{\infty}(X, E)^{\prime}$; if we define $u(\phi)$ for each $\phi \in \mathcal{K}(X)$ by $\langle y, u(\phi)\rangle=L(\phi \otimes y)$ for all $y \in E$, then $u$ is an $E_{w}^{\prime}$-valued bounded Radon measure. The transpose $u^{\prime}$ of $u$ is a linear map from $E$ into $M_{b}(X)$, the space of all bounded Radon measures on $X$. For every $y \in E$ there corresponds a unique regular Borel measure $\mu_{y}$ such that $\mu_{y}(B)=\left\langle u^{\prime}(y), \chi_{B}\right\rangle$, for all Borel subsets $B$ of $X$. There exists a continuous seminorm $p$ on $E$ and a constant $k>0$ such that $|L(f)| \leqq k\|f\|_{p}$ for all $f \in C_{\infty}(X, E)$. Hence $|\langle y, u(\phi)\rangle|=|L(\phi \otimes y)| \leqq k p(y)\|\phi\|_{\infty}$. Thus, the bounded Radon measure $u^{\prime}(y)$ has norm $\left\|u^{\prime}(y)\right\| \leqq k p(y)$, and the corresponding Borel measure $\mu_{y}$ is such that $\left|\mu_{y}(B)\right| \leqq\left\|\mu_{y}\right\| \leqq k p(y)$. This shows that, for a fixed Borel subset $B \subset X$, the map $y \mapsto \mu_{y}(B)$ belongs to $E^{\prime}$. Call this map $\mu(B)$. The set function $B \mapsto \mu(B)$, defined on the $\sigma$-ring of all Borel subsets of $X$ and with values on $E^{\prime}$, is countably additive. For any finite families $\left\{B_{i}\right\}_{i \in I}$ of disjoint Borel subsets of $X$, whose union is $X$, and $\left\{y_{i}\right\}_{i \in I}$ of elements of $E$ with $p\left(y_{i}\right) \leqq 1$ for each $i \in I$, we have

$$
\left|\sum_{i \in I}\left\langle y_{i}, \mu\left(B_{i}\right)\right\rangle\right| \leqq k .
$$

An $E_{w}^{\prime}$-valued bounded Radon measure $u$ on $X$ such that the corresponding set function $\mu$ satisfies (*) for some continuous seminorm $p$ on $E$ and some constant $k>0$ is said to have finite $p$-semivariation. On the other hand, following Dieudonné [2], an $E_{w}^{\prime}$-valued bounded 
Radon measure on $X$ is said to be $p$-dominated if there is a positive bounded Radon measure $\mu$ on $X$ such that $|\langle y, u(\phi)\rangle| \leqq \mu(|\phi|) p(y)$ for all $y \in E$ and $\phi \in \Re(X)$. The arguments contained in Singer [9] and Các [1] can be extended to prove the following:

Lemma. Let $u$ be an $E_{w}^{\prime}$-valued bounded Radon measure on $X$. The following are equivalent:

(a) $u$ is integral;

(b) $u$ is p-dominated, for some continuous seminorm $p$ on $E$;

(c) $u$ has finite p-semivariation, for some continuous seminorm $p$ on $E$.

We denote by $M_{b}\left(X, E^{\prime}\right)$ the vector space of all $E_{w}^{\prime}$-valued bounded Radon measures on $X$ which satisfy (a) or (b) or (c).

TheOREM 3. Let $C V_{\infty}(X, E)$ be a Nachbin space. Then $V M_{b}\left(X, E^{\prime}\right)$ is linearly isomorphic to $C V_{\infty}(X, E)^{\prime}$.

4. Bishop's generalized Stone-Weierstrass theorem [7]. If $A$ is a subalgebra of $C(X)$, a subset $K \subset X$ is said to be antisymmetric for $A$ if, for $f \in A$, the restriction $f \mid K$ being real-valued implies that $f \mid K$ is constant. Every antisymmetric set for $A$ is contained in a maximal one, and the collection $\mathfrak{K}_{A}$ of maximal antisymmetric sets for $A$ forms a closed, pairwise disjoint covering of $X$ (Glicksberg [3]). The following form of Bishop's generalized Stone-Weierstrass theorem is valid for Nachbin spaces ( $X$ is as in $\S 3$ ).

TheORem 4. Let $V \subset C^{+}(X)$ and let $A$ be a subalgebra of $C(X)$ such that every $g \in A$ is bounded on the support of every $v \in V$. Let $W$ be a vector subspace of $C V_{\infty}(X, E)$ which is an $A$-module. Then $\in C V_{\infty}(X, E)$ is in the closure of $W$ if and only if $f \mid K$ is in the closure of $W \mid K$ in $C V_{\infty}(K, E)$ for each $K \in \mathfrak{K}_{A}$.

If $E$ is $R$ or $C$ the hypothesis $V \subset C^{+}(X)$ can be strengthened to $V \leqq C^{+}(X)$. If $A$ is selfadjoint, the conclusion of Theorem 4 is that $W$ is localizable under $A$ in $C V_{\infty}(X)$ (see Definition 4, Nachbin [5]). Let $C V_{\infty}(X, E)$ be an $A$-module, where $A$ satisfies the hypothesis of Theorem 4 and its maximal antisymmetric sets are sets reduced to a point, (e.g., $C_{b}(X)$, the algebra of all bounded continuous complexvalued functions). Under this hypothesis the following spectral synthesis result holds.

TheOREM 5. Every proper closed A-submodule $W \subset C V_{\infty}(X, E)$ is contained in some closed $A$-submodule of codimension one in $C V_{\infty}(X, E)$ and is the intersection of all proper closed $A$-submodules of codimension one in $C V_{\infty}(X, E)$ which contain it. 
5. Dieudonné theorem for density in tensor products of Nachbin spaces [8]. Let $X$ and $Y$ be two completely regular Hausdorff spaces and $V$ and $W$ two directed families of weights on $X$ and $Y$ respectively. Let $V \times W$ denote the set of all functions $(x, y) \mapsto v(x) w(y)$ on $X \times Y$. Let $A$ be a locally convex topological algebra and let $E$ and $F$ be two locally convex spaces which are topological modules over $A$. Then $E \otimes{ }_{A} F$ is defined to be the quotient space $(E \otimes F) / D$, where $E \otimes F$ has the projective tensor product topology and $D$ is the closed linear span of the elements of the form $a u \otimes v-u \otimes a v$, where $a \in A$, $u \in E, v \in F$. If $f \in C V_{\infty}(X, E)$ and $g \in C W_{\infty}(Y, F)$, then $f \otimes_{A} g$ belongs to $C(V \times W)_{\infty}\left(X \times Y, E \otimes_{A} F\right)$, where $f \otimes_{A} g$ denotes the map $(x, y) \mapsto f(x) \otimes_{A} g(y)$.

THEOREM 6. The vector subspace of all finite sums of mappings of the form $f \otimes_{A} g$, where $f \in C V_{\infty}(X, E)$ and $g \in C W_{\infty}(Y, F)$, is dense in $C(V \times W)_{\infty}\left(X \times Y, E \otimes_{A} F\right)$.

\section{REFERENCES}

1. N. P. Các, Linear transformations on some functional spaces, Proc. London Math. Soc. (3) 16 (1966), 705-736.

2. J. Dieudonné, Sur le théorème de Lebesgue-Nikodym. V, Canad. J. Math. 3 (1951), 129-139. MR 13, 448.

3. I. Glicksberg, Bishop's generalized Stone-Weierstrass theorem for the strict topology, Proc. Amer. Math. Soc. 14 (1963), 329-333. MR 26 \#4165.

4. A. Grothendieck, Produits tensoriels topologiques et espaces nucléaires, Mem. Amer. Math. Soc. No. 16 (1955). MR 17, 763.

5. L. Nachbin, Weighted approximation for algebras and modules of continuous functions: Real and self-adjoint complex cases, Ann. of Math. (2) 81 (1965), 289-302. MR 31 \#628.

6. J. B. Prolla, Weighted spaces of vector-valued continuous functions, Ann. Mat. Pura Appl. (to appear).

7. - Bishop's generalized Stone-Weierstrass theorem for weighted spaces, Math. Ann. 191 (1971), 283-289.

8. - The weighted Dieudonnê theorem for density in tensor products, Nederl. Akad. Wetensch. Proc. Ser. A. 74 = Indag. Math. 33 (1971), 170-175.

9. I. Singer, Sur les applications linéaires intégrales des espaces de fonctions continues. I, Rev. Math. Pures Appl. 4 (1959), 391-401. MR 22 \#5883.

10. W. H. Summers, A representation theorem for biequicontinuous completed tensor products of weighted spaces, Trans. Amer. Math. Soc. 146 (1969), 121-131. MR 40 \#4748.

UNIVERSity OF ROchester, Rochester, New YoRK 14627 